\title{
Image Steganography Analysis Based on Deep Learning
}

\author{
Vijay Kumar*, Pankaj Rao, Ashish Choudhary \\ Computer Science and Engineering Department, National Institute of Technology, Hamirpur, H.P. 177005, India
}

Corresponding Author Email: vijaykumarchahar@nith.ac.in

https://doi.org/10.18280/rces.070101

Received: 10 February 2020

Accepted: 16 March 2020

\section{Keywords:}

steganography, embedding rate, convolutional neural network, global information, steganalysis, co-occurrence matrix, multi-class, feature learning

\begin{abstract}
Despite the fact that steganalysis has grown quickly lately, it despite everything faces numerous troubles and difficulties. This paper proposed a steganalysis based on deep learning technique. The convolutional neural network method is used for steganalysis approach. The steganalysis approach utilizes the deep features extracted from the stego image. The approach uses global information for feature learning. The steganalysis is used to recognize the various kinds of steganalysis calculations. This paper also proposed a steganalysis based on low embedding rate images and multi-class steganography. The study on image steganogrphy using deep learning is discussed with their pros and cons.
\end{abstract}

\section{INTRODUCTION}

With a fast improvement of data innovation and network technologies, electronic media gets a significant transporter for military, business, and different associations just as people to acquire and transmit data [1-3]. At present, an automated conversation in the online network is helpless against the risk of spying and vicious activities [4]. Hence, the security concerns should be given more consideration. The customary arrangement utilizes encryption innovation to change over the data to ciphertext for transmission [5]. The main advantages of this method are that it is easily recognized by the attackers. It does not provide any type of security when data is transmitted from one place to another. Therefore, steganography, which is described by "camouflage" in the transmission of data, has pulled in increasingly more consideration [6].

Steganography is hiding the information into the cover image and transmitted from one person to another in a secure manner. The secret information is hiding into the cover image so that no one can see this through naked eyes or even cannot be recognized by the attackers. Let's take the example to understand steganography more deeply. Vijay and Ashish are two good friends and lived in the Himgiri Boys Hostel of NITH in two different rooms. They planned to escape from the hostel and chill outside. Both of them are under the care of hostel warden. According to the hostel rules and regulations, hostel warden supervises their communication. Therefore, they will not be talk in plaintext. There are two choices to communicate either communicate through encryption technique or message is shielded in other objects [7]. The most desirable method is to hide the secret information in some other objects. Steganography is widely used for this task. Secret conversations within the representatives of Al Qaeda were reported by some news agency in United states such as $\mathrm{CNN}$. The gang of Bin laden attacks the particular location of somewhere, instructions related to actions and other messages are hidden in various websites on online network. There are many real-life scenarios related to the Steganography.
There is a need to analyze the deep learning based steganography tecghniques.

This paper tries to form the new idea to learn feature expression through steganalysis. The main contributions of this paper are as follows:

(1) Convolutional Neural Network (CNN) method is used for steganalysis approach. Steganalysis technique is used to analyze the feature expression. In this method, firstly design the model, i.e., CNN and implemented in such a manner that it can efficient and effective to capture the numerical attributes such as neighborhood relationship. This technique is not used the same as the past strategy. It incorporates the element extraction and grouping module It makes an intelligent systems prototype. Detection realization of the model can also be improved.

(2) Comprehensive intelligence based steganalysis method is studied. This is an advanced version related to the deep learning technique. It uses global information to learn the feature expression more efficiently and enhance the throughput of the model. It can work on $\mathrm{CNN}$ model and improve them. The feature expression is grasped productively.

(3) The low installing amount steganalysis highlights learning technique is discussed. The less installing rate encrypted images is a problem in the steganalysis because it cannot be detected easily. To overcome this problem, the representation grasping is improved on small installing rate images by relocating the knowledge gained from the large embedded rate images.

(4) The multiple classes steganography method is discussed. From the current research, we know that the different types of steganalysis algorithms use the same algorithms to construct the detectors. To construct the detectors, the edge information is used to produce the dense images. There are different types of steganography algorithms in real-life scenarios. It is also difficult to know for the steganalysts that steganography algorithm is used by the users. So, it is important to know all types of algorithms.

The remainder of this paper is organized as follows: Section 2 presents the steganalysis based on convolutional neural 
networks. The global information based steganalysis is presented in Section 3. Section 4 presents the embedding rate based steganalysis. Section 5 presents the steganalysis on multiclass steganography. The discussions are depicted in Section 6. The concluding remarks are drawn in Section 7.

\section{CONVOLUTION NEURAL NETWORK BASED STEGANALYSIS}

George Mason University in Virginia started the study on the topic of steganalysis in 1990s. After that, this topic magnetized the number of universities such as New York State University, Purdue University, etc. Steganalysis and steganography research have developed in the last few years. Steganography brings new difficulties to the steganalysis. The advance version of steganography creates new problems for steganalysis. The new concepts are developed by the advance steganography that is difficult to steganalysis. To overcome this difficulty, the in-depth learning feature extraction techniques is used. Feature expression enhances the throughput and detection of the secret message hidden into the cover image. In historic time, this feature expression is done by the human manually. This process takes too much time and human faces difficulties to extract the feature expression [810]. The steganalysis is rapidly developing day by day and numbers of new features are merge into it. It also makes the extraction of feature expression more complex and difficult. Adaptive steganography algorithms have higher complicated numerical properties.

To overcome the feature expression problem, in-depth learning technique is introduced. In this method, the human cannot manually extract the feature expression. It can be done by the $\mathrm{CNN}$ trained model. In this, $\mathrm{CNN}$ model is in combination with steganalysis properties and makes the newly trained network that is used to extract the feature expression easily.

\subsection{Steganalysis feature learning framework}

In this convolutional neural network, the complex feature expression is presented by the multiple convolutional layers so that the extraction of the feature expression is easily recognized. the model which can efficiently and effectively learn the feature expression automatically through training. At present, steganography calculations are increasingly mind boggling measurable qualities, i.e., neighborhood connection. Because of the less exact picture measurable model and the versatile steganography shrouds the data into the picture locale with commotion and it is hard to structure such a model. In historic time, the feature expression is manually done by the human which faces more difficulty. So the neighborhood correlation is done on a deep learning model to design an effective model that is inspired by the traditional method of steganalysis. That's why the CNN based steganalysis model is suggested. In this model, a large amount of data is captured by $\mathrm{CNN}$ to find the correlation between the adjacent pixels of the images, which is much more important for steganalysis. Different layers of CNN namely pooling, weight sharing additionally diminish the preparation parameters of the model. It can deal with the huge size and countless pictures. The appropriate model is designed that can effectively extract the feature expression for the given dataset [11-13]. Figure 1 shows the feature learning framework using CNN.

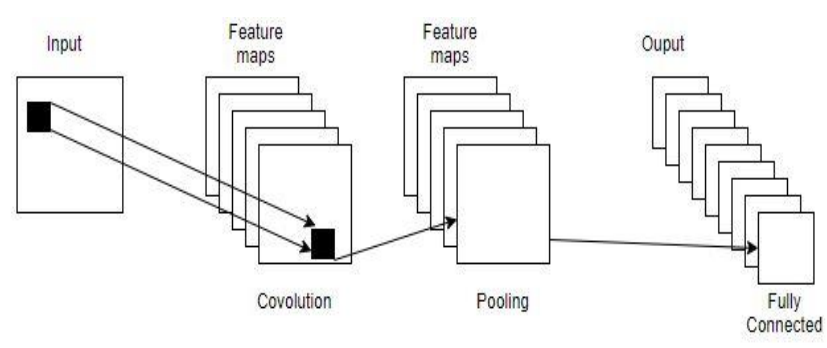

Figure 1. Steganalysis feature learning framework using $\mathrm{CNN}$

\section{GLOBAL INFORMATION CONSTRAINTS BASED STEGANALYSIS}

CNN model is used to automatically learns the feature expression. CNN introduced the statistical global information and give the better feature learning. Global information is applied on the entire image in the steganalysis. Steganography is basically adding the noise to the image, but the noise is relatively small as compared to the content of the data into the image. Steganalysis needed two aspects of information. The first one is the native intelligence and the second is global information. Both the aspects are extremely important for steganalysis [14].

The researchers in this field note very late the significance of the global steganalysis image figures. It also tends to be a issue in function design. CNN's earlier approach is useful for defining the relationship among markers of the local area. Within the CNN model, the pooling layer fuses the localized image details. There is to be the multi-layer pooling process in the new solution. As contrasted with the earlier universal systematic approach, i.e., histogram and measure of cooccurrence. The various classes in histogram and preoccurrence equation can be determined from the entire plot. we concentrate only on how can develop the global information techniques [15].

\subsection{Steganalysis based upon global information constraint}

To obtain the accurate result, one should know about the major quantitative data in steganalysis. This technique leaves the impression of transition learning for special mention. It proposes the global statistical knowledge model based on the CNN. To obtain the better feature expression, one can use the global information model. Transfer learning is used to transfer the knowledge in tasks. The success point is that the first two tasks are related to each other. One task helps the other task and easily recognized itself. In this method, the long stream information is presented in 3D CNN model. The importance of this is to improve the throughput of the global statistical information model by transfer learning [16-19]. Figure 2 shows the global information based steganalysis.

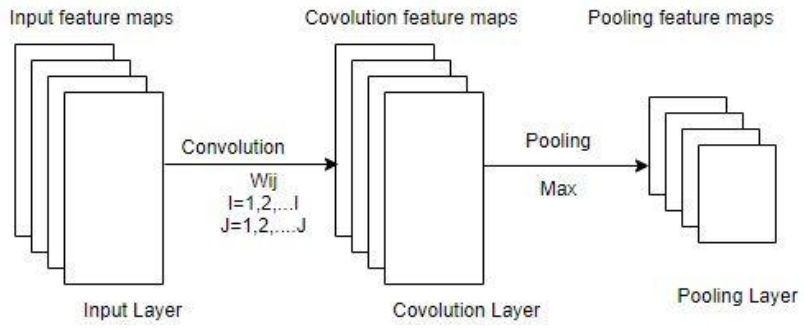

Figure 2. Steganalysis based upon global information 


\section{LOW EMBEDDING RATE BASED STEGANALYSIS}

The in-depth CNN and global CNN model are based on learning and empirical knowledge, respectively. Both the model focuses on the learning. The secret information is concealed in the cover image. Steganographic disturbance is implemented by steganographic procedures into the pictures. The difference between the image after and before is indeed very low and the distinction between different features of the image can easily be lost. The hardest challenge is managing photos at low encoding speeds. When the amount of information hidden in the cover picture decreases then the steganalysis complexity increases. Adaptive steganography performs the reduced encoding volume and difficult to detect [20].

\subsection{Analysis of content adaptive steganography}

The content-adaptive steganography algorithm is one of the important and secure steganography algorithms. It uses the complex statistical information in the texture region of the images. The images having low embedding rate are very hard

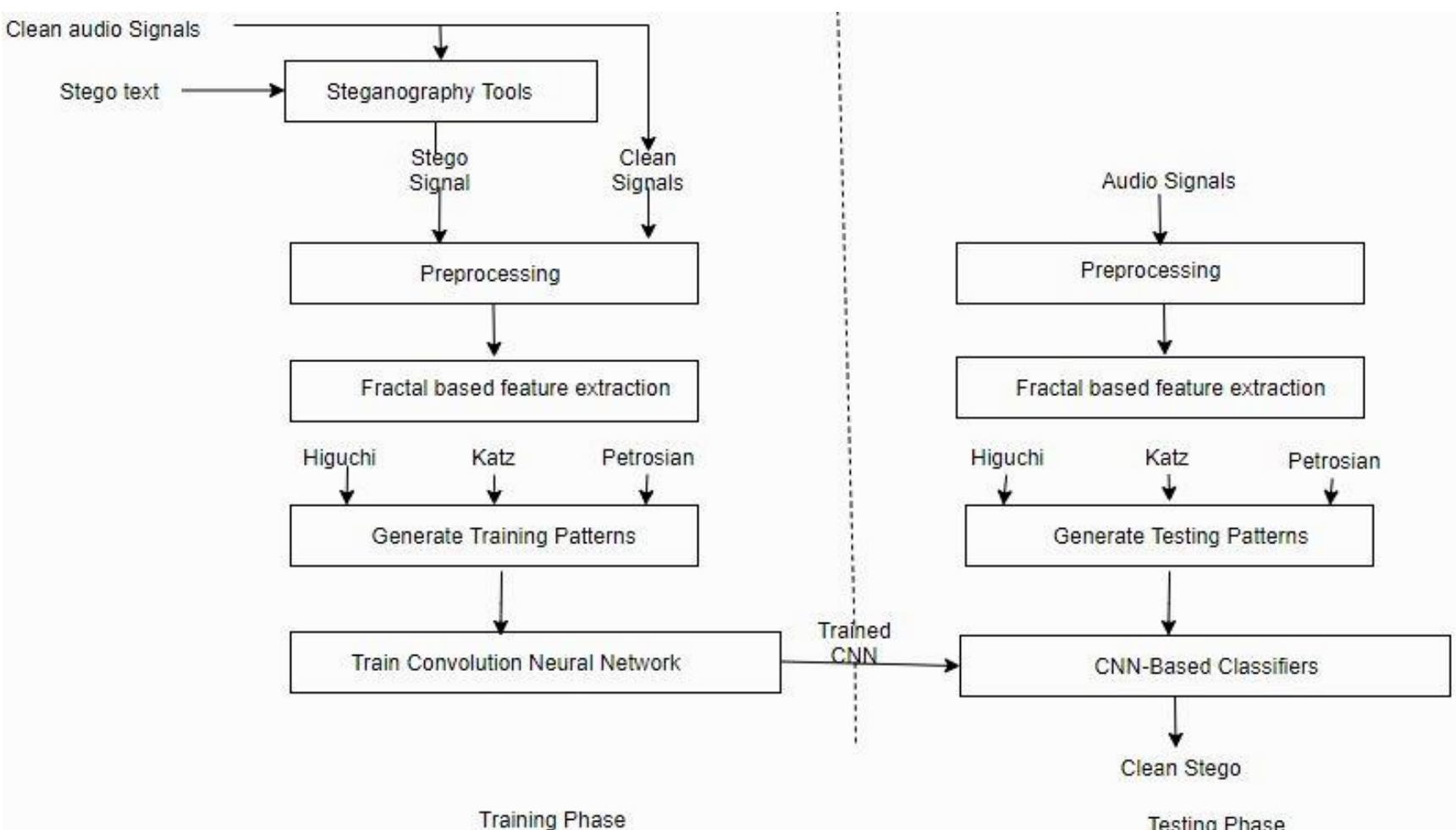

Figure 3. Steganalysis based upon Low embedding rate to identify as compared with a high encoding amount. First, the information is embedded in the texture region of the image [21]. Figure 3 shows the embedding position of the information after embedded the information into the cover images.

The adaptive steganography can be hard to identify at a low embedding rate. It is hard for several scientists to identify the material-adaptive steganography at a small encoding level. Also, there are a few widely known stuffs found on the numerical attributes of the image in the different encoding level. The derivable information should be used in large encoding level images so that it can be easily identified by the small encoding level images. The transfer learning is used for low encoding level steganalysis. This approach varies from the previous method as it manages the embedded images independently. The performance of image detection is to enhance with a low embedding rate and the pre-trained model without the additional features or information. This method contains two tasks. The first module identifies photos at low encoding levels as well as the second module identifies images at large encoding levels.

\section{MULTI-CLASS STEGANALSIS}

The steganalysis for multi-class steganography is discussed. The different forms of steganalysis implementations have the same algorithms to create the sensors. If we have more edge information, than it can help us to make a more efficient model. The real-life situations have various kinds of steganography implementations. It is also difficult to know for the steganalysis that which steganography algorithm is used by the users.

There have been few experiments onto the design of the detector in the study of image steganalysis, which help us to detect the hidden messages in the cover images [22-24]. First, the steganographic algorithms are applied to the original images and then the extraction of feature learning is taking place. The two researchers Pevny's and Ffidrich's uses four JPEG domain steganographic algorithms. Two SVM classifiers are educated. These two classifiers help us to find the difference between the original image and the stego images. In the second method, it is similar to the first method till extraction and then the steganalysis of multi-class steganography is done. It means multi-classifiers are produced to find the difference between the original and the stego images. Till now, much more work has to be done in the field of the steganalysis. 


\subsection{Steganalysis framework based on multi-task CNN}

The structure for steganalysis is dependent upon multi-task CNN. First of all, the mega-steganographic protocol is to be developed in this context. As well as the prototype is then educated using mega-task training. In the final step of this method, the steganographic algorithms are used to encode the messages into the images. So, in this way it gives high efficiency to detect at the final step [8, 23-25]. Figure 4 shows the steganalysis framework based on multi task CNN.

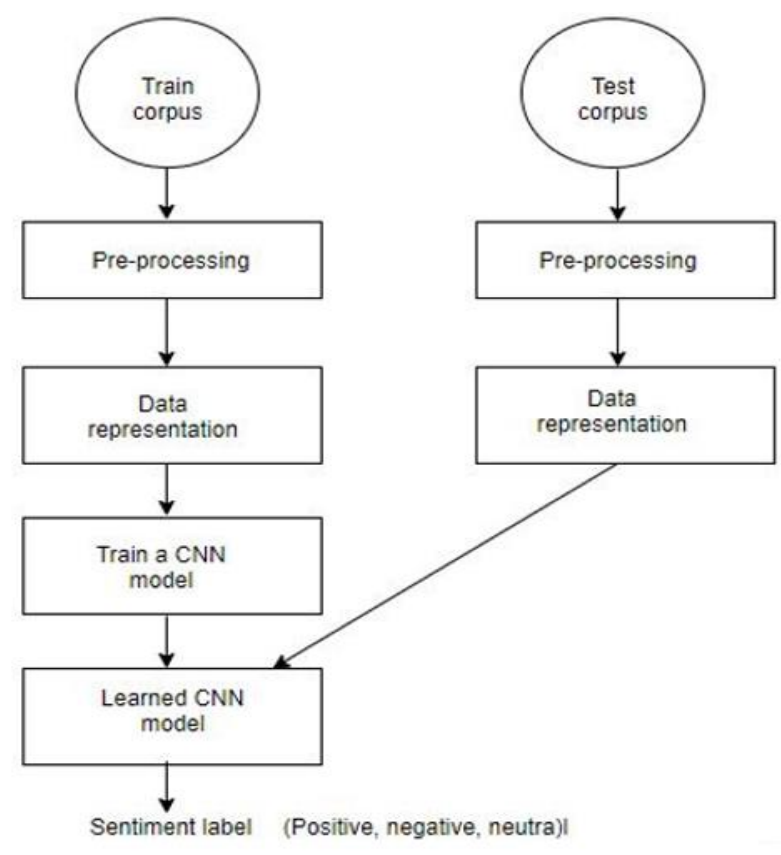

Figure 4. Steganalysis framework based on multi-task CNN

\section{DISCUSSION}

In the in-depth learning method, the main focus is to feature learning. In-depth leaning method automatically learns the feature expression. They used statistical characteristics such as neighborhood correlation for steganalysis. The global information constraints are used for analysis of steganography. The global information is much more useful than the traditional method. The pooling combines the local picture knowledge to use of knowledge across a wide range. There are some limitations of global information and to overcome these limitations. The small encoding level enciphered photos is a problem in the steganalysis because it cannot be detected easily. The function learning on small encoding level images is used for relocating the knowledge acquired from the heavy encoding level images. The different types of steganalysis protocols use identical algorithms to build the identifiers. To construct the detector, the edge information is used for dense images. There are different types of steganography algorithms in real-life scenarios. It is also difficult to know for the steganalysts which steganography algorithm is used by the users. So, it is important to know all types of algorithms.

\section{CONCLUSIONS}

The Internet and digital media become an effective method that saves human money and time. And on the other side, it is illegally used by terrorist gangs and other organizations. The main pros for these technologies are security. With the help of steganography, the terrorist gangs and other organizations plan their mission without knowing anyone in the entire world. This paper introduced the deep learning, global information, low embedding rate, and multi-class steganalysis techniques. There is a number of cons and pros of these techniques which we are using in this paper. With the help of in-depth learning and global information, feature learning becomes easy. The stego and the original images look similar so that no one can recognize which one is the stego image and the original image. And the pros are that the computation is to complex.

\section{REFERENCES}

[1] Johnson, N.F., Jajodia, S. (1998). Exploring steganography: Seeing the unseen. Computer, 31(2): 2634. https://doi.org/10.1109/MC.1998.4655281

[2] Fridrich, J., Goljan, M., Du, R. (2001). Detecting LSB steganography in color, and Gray-scale images. IEEE Multimedia, $8(4)$ : 22-28. https://doi.org/10.1109/93.959097

[3] Cheddad, A., Condell, J., Curran, K., Mc Kevitt, P. (2010). Digital image steganography: Survey and analysis of current methods. Signal Processing, 90(3): 727-752. https://doi.org/10.1016/j.sigpro.2009.08.010

[4] Zhang, L., Song, M., Liu, Z., Liu, X., Bu, J., Chen, C. (2013). Probabilistic graphlet cut: Exploiting spatial structure cue for weakly supervised image segmentation. In Proceedings of the IEEE Conference on Computer Vision and Pattern Recognition, pp. 1908-1915. https://doi.org/10.1109/CVPR.2013.249

[5] Cachin, C. (2004). An information-theoretic model for steganography. Information and Computation, 192(1): 41-56. https://doi.org/10.1016/j.ic.2004.02.003

[6] Marvel, L.M., Boncelet, C.G., Retter, C.T. (1999). Spread spectrum image steganography. IEEE Transactions on Image Processing, 8(8): 1075-1083. https://doi.org/10.1109/83.777088

[7] Artz, D. (2001). Digital steganography: Hiding data within data. IEEE Internet Computing, 5(3): 75-80. https://doi.org/10.1109/4236.935180

[8] Han, J., Zhang, D., Hu, X., Guo, L., Ren, J., Wu, F. (2014). Background prior-based salient object detection via deep reconstruction residual. IEEE Transactions on Circuits and Systems for Video Technology, 25(8): 1309-1321. https://doi.org/10.1109/TCSVT.2014.2381471

[9] Han, J., Ngan, K.N., Li, M., Zhang, H.J. (2005). Unsupervised extraction of visual attention objects in color images. IEEE Transactions on Circuits and Systems for Video Technology, 16(1): 141-145. https://doi.org/10.1109/TCSVT.2005.859028

[10] Zhang, L., Xia, Y., Ji, R., Li, X. (2014). Spatial-aware object-level saliency prediction by learning graphlet hierarchies. IEEE Transactions on Industrial Electronics, 62(2): https://doi.org/10.1109/TIE.2014.2336602

1301-1308.

[11] Han, J., Zhang, D., Cheng, G., Guo, L., Ren, J. (2014). Object detection in optical remote sensing images based on weakly supervised learning and high-level feature learning. IEEE Transactions on Geoscience and Remote Sensing, $\quad$ 53(6):

3325-3337. 
https://doi.org/10.1109/TGRS.2014.2374218

[12] Luo, W.Q., Huang, F.J., Huang, J.W. (2010). Edge adaptive image steganography based on LSB matching revisited. IEEE Transactions on Information Forensics and Security, 5(2): 201-214. https://doi.org/10.1109/TIFS.2010.2041812

[13] Zhang, L., Hong, R., Gao, Y., Ji, R., Dai, Q., Li, X. (2015). Image categorization by learning a propagated graphlet path. IEEE Transactions on Neural Networks and Learning Systems, 27(3): 674-685. https://doi.org/10.1109/TNNLS.2015.2444417

[14] Girdhar, A., Kumar, V. (2019). A reversible and affine invariant $3 \mathrm{D}$ data hiding technique based on difference shifting and logistic map. Journal of Ambient Intelligence and Humanized Computing, 10(12): 49474961. https://doi.org/10.1007/s12652-019-01179-4

[15] Girdhar, A., Kumar, V. (2017). Comprehensive survey of 3D image steganography techniques. IET Image Processing, 12(1): 1-10. https://doi.org/10.1049/ietipr.2017.0162

[16] Lin, C.C., Tsai, W.H. (2004). Secret image sharing with steganography and authentication. Journal of Systems and $\quad$ Software, $\quad 73(3)$ : 405-414. https://doi.org/10.1016/S0164-1212(03)00239-5

[17] Hussain, M., Hussain, M. (2013). A survey of image steganography techniques. International Journal of Advanced Science and Technology, 54: 113-124.

[18] Han, J.W., Ji, X., Hu, X.T., Zhu, D.J., Li, K.M., Jiang, X., Cui, G.B., Guo, L., Liu, T.M. Representing and retrieving video shots in humancentric brain imaging space. IEEE Trans. Image Process, 22(7): 2723-2736. https://doi.org/10.1109/TIP.2013.2256919

[19] Zhang, T., Guo, L., Li, K.M., Jing, C.F., Yin, Y., Zhu, D.J., Cui, G.B., Li, L.J., Liu, T.M. (2012). Predicting functional cortical ROIs via DTI-derived fiber shape models. Cerebral Cortex, 22(4): 854-864. https://doi.org/10.1093/cercor/bhr152

[20] Zhang, L., Gao, Y., Xia, Y., Dai, Q., Li, X. (2014). A fine-grained image categorization system by celletencoded spatial pyramid modeling. IEEE Transactions on Industrial Electronics, 62(1): 564-571. https://doi.org/10.1109/TIE.2014.2327558

[21] Zhang, D., Meng, D., Han, J. (2016). Co-saliency detection via a self-paced multiple-instance learning framework. IEEE Transactions on Pattern Analysis and Machine Intelligence, 39(5): 865-878. https://doi.org/10.1109/TPAMI.2016.2567393

[22] Zhang, L., Gao, Y., Xia, Y., Lu, K., Shen, J., Ji, R. (2013). Representative discovery of structure cues for weaklysupervised image segmentation. IEEE Transactions on Multimedia, 16(2): 470-479. https://doi.org/10.1109/TMM.2013.2293424

[23] Zhang, D.W., Han, J.W., Li, C., Wang, J.D., Li, X.L. (2016). Detection of co-salient objects by looking deep and wide. International Journal of Computer Vision, 120(2): 215-232. https://doi.org/10.1007/s11263-0160907-4

[24] Cheng, G., Zhou, P., Han, J. (2016). Learning rotationinvariant convolutional neural networks for object detection in VHR optical remote sensing images. IEEE Transactions on Geoscience and Remote Sensing, 54(12): 7405-7415. https://doi.org/10.1109/TGRS.2016.2601622

[25] Dumitrescu, S., Wu, X., Wang, Z. (2002, October). Detection of LSB steganography via sample pair analysis. In International Workshop on Information Hiding, pp. 355-372. https://doi.org/10.1109/TSP.2003.812753 